$>$ Les microtubules sont des fibres du cytosquelette formées par l'assemblage d'hétérodimères d' $\alpha$ - et de $\beta$-tubuline. Ils contribuent à l'établissement de la forme des cellules et de leur polarité, ainsi qu'à leur mobilité. Ils jouent aussi un rôle important dans le transport intracellulaire et dans la division cellulaire. Le réseau microtubulaire s'adapte constamment aux besoins de la cellule. Il peut être constitué de microtubules très dynamiques ou d'autres plus stables. Pour moduler dans l'espace et le temps les différentes fonctions de ces fibres, de nombreuses modifications post-traductionnelles réversibles de la tubuline sont mises en jeu, à l'origine de ce qui est maintenant appelé le « code tubuline». Dans cette revue, nous nous intéresserons au rôle de deux modifications caractéristiques des microtubules stables: I'acétylation et la détyrosination de l' $\alpha$-tubuline. Nous discuterons également de l'implication de leur dérégulation dans certaines pathologies. <

Les microtubules sont des constituants importants du cytosquelette. Ce sont des tubes creux formés par l'association de dimères d' $\alpha$-tubuline et de $\beta$-tubuline. Ces dimères sont assemblés en protofilaments. En règle générale, treize protofilaments s'alignent latéralement pour constituer la paroi des microtubules. Les microtubules sont des polymères orientés, avec une extrémité présentant la sous-unité $\beta$ de la tubuline, appelée « extrémité plus », et une extrémité qui se termine par la sous-unité $\alpha$, appelée «extrémité moins 》 (Figure 1). Ils s'allongent ou se raccourcissent par l'incorporation ou la libération de dimères de tubuline, l'incorporation étant plus rapide du côté «plus»de la fibre. Les phases d'allongement, appelées phases de croissance, peuvent être suivies de pauses ou de phases de dépolymérisation rapides, appelées « catastrophes », parfois interrompues par un redémarrage de l'assemblage, dénommé «sauvetage ». L'ensemble de

\section{Déchiffrage du code tubuline}

\section{Le voile se lève}

sur le rôle de l'acétylation et de la détyrosination

\author{
Karin Sadoul, Clotilde Joubert, Sophie Michallet, \\ Elsie Nolte, Lauralie Peronne, Sacnicté Ramirez- \\ Rios, Anne-Sophie Ribba, Laurence Lafanechère
}

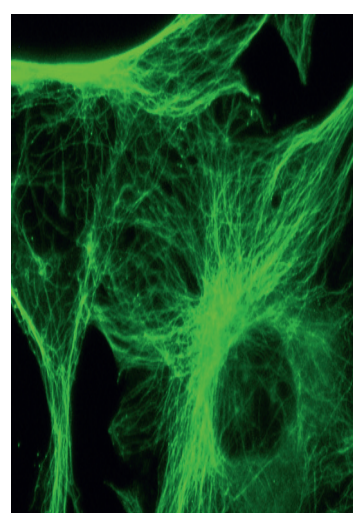

Régulation et pharmacologie du cytosquelette, Institut pour l'avancée des biosciences, Université Grenoble Alpes ; Inserm U 1209 ; CNRS 5309, Allée des Alpes, 38700 La Tronche, France.

laurence.lafanechere@univgrenoble-alpes.fr karin.sadoul@univ-grenoblealpes.fr

ces événements participe à l'instabilité dynamique des microtubules, une propriété intrinsèque de ces fibres (Figure 1 ).

Le diamètre large des microtubules ( $25 \mathrm{~nm}$ ) leur confère une rigidité beaucoup plus importante que celle des filaments d'actine. Leurs propriétés structurales et mécaniques les destinent à des fonctions multiples dans la cellule (Figure 2). Ils forment le fuseau mitotique, qui permet de répartir équitablement les chromosomes dans les cellules filles à l'issue de la mitose ; ils contrôlent la forme des cellules et se réorganisent lors des modifications nécessaires à leur différenciation, leur polarisation ou leur migration. Les microtubules sont aussi des éléments essentiels des cils et des flagelles dans lesquels ils adoptent une organisation particulière en se répartissant en doublets et en triplets. Enfin, ils servent de rails pour acheminer des vésicules, des protéines ou des organelles au sein de la cellule.

Pour accomplir ces tâches multiples, le réseau microtubulaire est fortement diversifié. Cette diversité résulte d'une part de leur composition en isoformes de tubuline. Sept isoformes principales de la sous-unité $\alpha$ et dix de la sous-unité $\beta$ de la tubuline sont exprimées dans les cellules humaines (pour une comparaison de leurs séquences voir [1]). D'autre part, les dimères de tubuline, libres ou assemblés en microtubules, peuvent être soumis à plusieurs modifications posttraductionnelles à l'origine de ce qui est appelé le « code tubuline ». Cette appellation a été établie par analogie avec le «code histone » qui est déchiffré par des facteurs de transcription et d'autres protéines liant la chromatine. De manière similaire, le code tubuline est interprété par les protéines qui s'associent et interagissent avec les microtubules, souvent dénommées MAP (pour microtubule-associated 


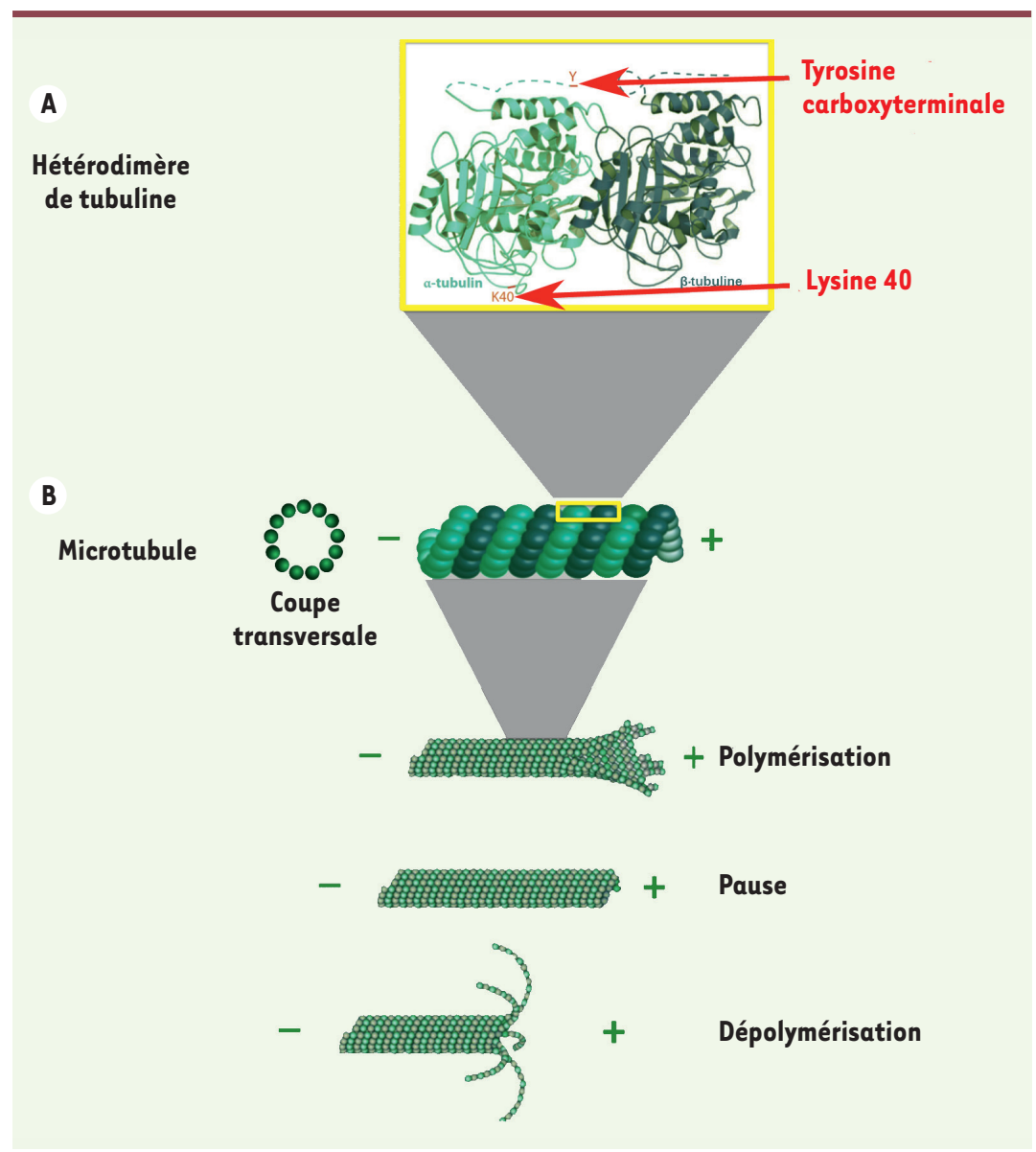

Figure 1. Composition et dynamique des microtubules. A. Représentation de l'hétérodimère d' $\alpha$ - et de $\beta$-tubuline, avec en rouge les sites d'acétylation et de détyrosination. B. Schéma d'un microtubule présentant les phases générant «l'instabilité dynamique».

manière aléatoire, lorsqu'elle diffuse dans la lumière du microtubule : plus le microtubule sera stable, plus il accumulera cette modification. Le degré d'acétylation d'un microtubule peut ainsi être considéré comme une sorte d'horodatage de sa durée de vie [6]. L'aTATl accède à la lysine 40 de la tubuline par les deux extrémités du microtubule ainsi qu'au travers de brèches dans sa paroi [7]. L'accès de l'enzyme est aussi facilité lorsque les microtubules sont en contact avec la membrane cellulaire, dans les domaines recouverts de clathrine où l' $\alpha$ TATl est recrutée par AP-2 (adaptor protein complex 2), un adaptateur de la clathrine $[8](\rightarrow)$.

$\rightarrow$ Voir la Nouvelle de G. Montagnac et P. Chavrier, $m / s n^{\circ} 2$ février 2014, page 130

\section{Les tubuline déacétylases}

L'HDAC6 (histone deacetylase 6) [9] et

proteins). Les nombreux types de modifications post-traductionnelles de la tubuline ont été revus en détail récemment [2]. Le présent article porte plus particulièrement sur l'état des connaissances concernant deux modifications très étudiées de la tubuline $\alpha$, caractéristiques des microtubules stables: I'acétylation de la lysine 40 et la détyrosination de l'extrémité carboxyterminale (Figure 1-3).

\section{La (dé)acétylation et ses enzymes}

L'acétylation d'une lysine est à l'origine une marque épigénétique décrite pour les histones. L'acétylation de l' $\alpha$-tubuline sur sa lysine en position 40 a été le premier exemple de cette modification post-traductionnelle détecté sur une protéine cytoplasmique [3]. L'acétylation de plusieurs autres lysines des tubulines $\alpha$ et $\beta$ a ensuite été décrite, mais leurs fonctions restent encore mal connues (pour revue, voir [1]).

\section{La tubuline acétyltransférase}

L'acétylation est catalysée par l'aTATI (alpha tubulin acetyl transferase 1) qui transfère le groupe acétyle du cofacteur acétyl-coenzyme A sur l'amine epsilon de la lysine en position 40 [4]. La conséquence directe de cette modification pour la protéine est la perte d'une charge négative et le gain d'une masse de 42 daltons (Figure 3A). Cette modification se fait à l'intérieur du cylindre microtubulaire, là où la lysine 40 est exposée [5]. L' $\alpha$ TATl acétyle les sous-unités $\alpha$ de la tubuline de la Sirt2 (silencing information regulator 2) [10] assurent la réversibilité de l'acétylation de la tubuline (Figure 3A). HDAC6 est une déacétylase de classe II qui dépend du zinc. Sirt2 appartient à la famille des sirtuines, ou déacétylases de classe III, qui dépendent du cofacteur $\mathrm{NAD}^{+}$(nicotinamide adénine dinucléotide). L'activité de Sirt2 est donc fortement liée à l'état métabolique de la cellule: plus la glycolyse produit d'énergie, moins le $N A D^{+}$, réduit en $N A D H$, est disponible pour la déacétylation par Sirt2. L'équilibre énergétique de la cellule contrôle donc le taux d'acétylation de la tubuline, que ce soit lors de l'acétylation (disponibilité d'acétyl-CoA) ou lors de la déacétylation (disponibilité du NAD+).

Le substrat favori de I'HDAC6 est le dimère de tubuline, et non pas le polymère [11]. Bien que I'HDAC6 soit la tubuline déacétylase majeure, il existe une sous-population de microtubules périnucléaires plus spécifiquement déacétylés par Sirt2 dans les cellules en interphase [12]. Lors de la mitose, Sirt2 co-localise avec la protéine Furry (Fry), qui réprime son activité au niveau des microtubules fortement acétylés du fuseau mitotique puis du corps intermédiaire. Il est important de noter que la lysine 40 de la tubuline $\alpha$ peut aussi être tri-méthylée par l'histone 


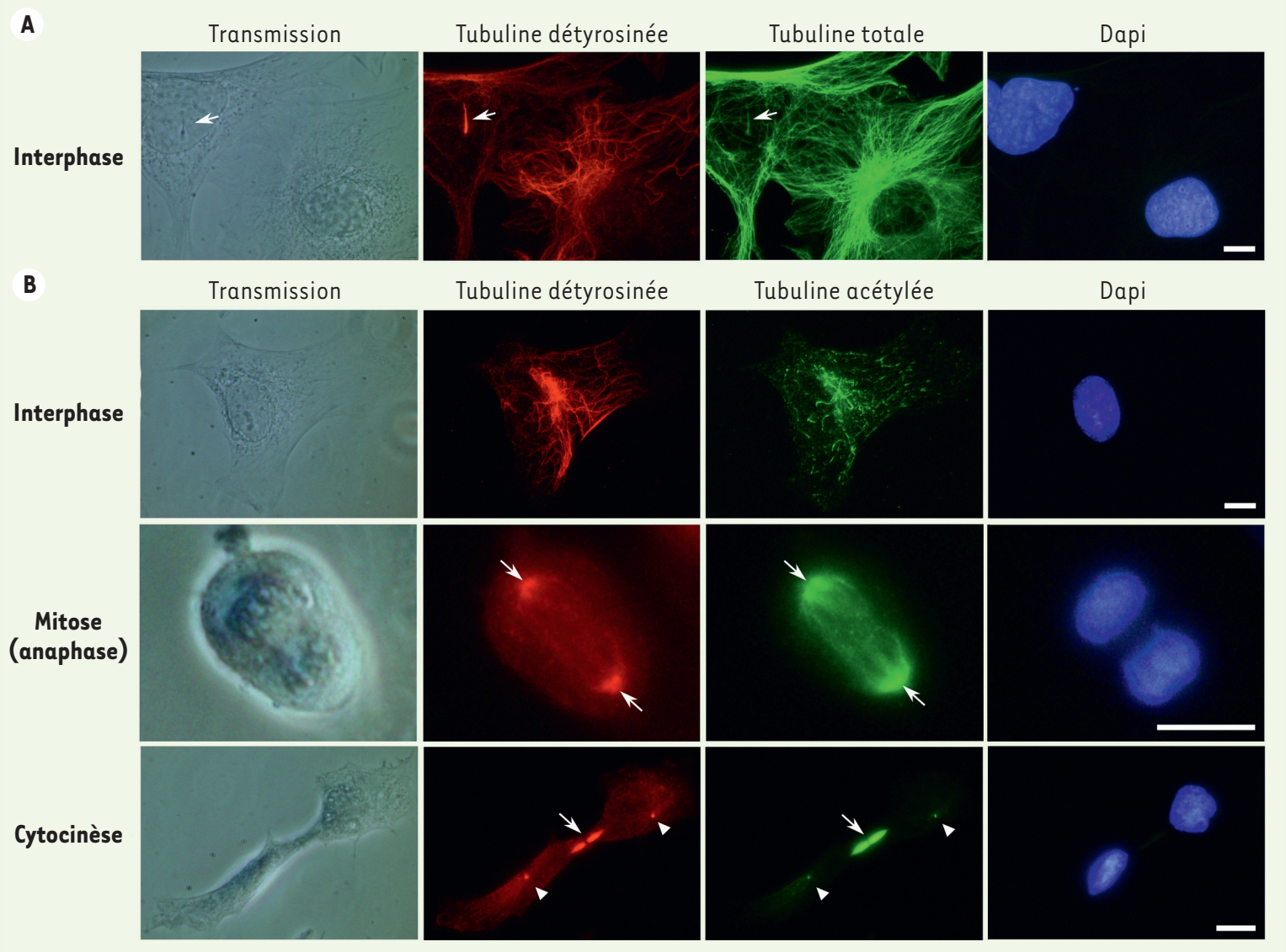

Figure 2. L'organisation des microtubules dans les cellules. A. Détection par immunofluorescence des microtubules détyrosinés (rouge) et totaux (vert) dans les cellules MEF (mouse embryonic fibroblasts) en interphase. Seule une partie du réseau microtubulaire ainsi que le cil primaire (flèche) sont marqués par l'anticorps qui reconnaît spécifiquement l'extrémité C-terminale détyrosinée de l' $\alpha$-tubuline. Remarque : le cil primaire est également acétylé [65], ce qui n'est pas illustré ici (barre, $10 \mu \mathrm{m}$ ). B. Détection par immunofluorescence des microtubules détyrosinés (rouge) et acétylés (vert) dans les cellules MEF en interphase, en mitose et pendant la cytocinèse. À noter qu'en interphase, on n'observe pas toujours une superposition du marquage tubuline acétylée et tubuline détyrosinée. En revanche, les extrémités (-) du fuseau mitotique (flèches), le corps intermédiaire (flèches) et les centres organisateurs des microtubules (têtes de flèche) sont marqués par les deux anticorps (barre, $10 \mu \mathrm{m}$ ).

méthyltransférase SETD2 (SET domain containing 2). Cette méthylation est observée dans le fuseau mitotique où elle est maintenue jusqu'à la formation du corps intermédiaire; elle est essentielle pour une mitose et cytocinèse correctes [13]. II reste à comprendre comment s'organisent ces évènements, c'est-à-dire si la tri-méthylation et l'acétylation co-existent au sein des microtubules mitotiques ou si Sirt2 et Furry doivent se dissocier pour permettre la déacétylation de la lysine 40 par Sirt2 et ensuite sa triméthylation par SETD2.

\section{Rôles de l'acétylation}

L'acétylation de la lysine 40 est en quelque sorte une marque du « code tubuline ». Existe-t-il des protéines capables de « lire » spécifiquement cette marque sur la tubuline, similairement au «code histone » où les lysines acétylées sont «lues » par des protéines nucléaires à bromodomaines?
Une protéine cytoplasmique à bromodomaine, dénommée tcBDF3 (Trypanosoma cruzi bromodomain factor 3) [14], a été identifiée chez les trypanosomes. Elle se lie à l' $\alpha$ tubuline acétylée, suggérant que les marques d'acétylation, qu'elles soient cytoplasmiques ou nucléaires, peuvent être lues par des protéines à bromodomaines spécifiques. L'acétylation des microtubules est plus une conséquence qu'une cause de leur stabilisation. Les souris déficientes en HDAC6, qui présentent une hyper-acétylation des microtubules dans tous les tissus, de même que les souris dépourvues d'expression d' $\alpha$ TATl (caractérisées par une absence presque complète de microtubules acétylés) sont viables et ne présentent que peu d'altérations physiologiques. Ceci indique que l'acétylation de la tubuline est plus particulièrement engagée dans la régulation fine des processus impliquant le cytosquelette. Par exemple, 
le transport vésiculaire ainsi que le transport des mitochondries et du réticulum endoplasmique le long des microtubules est plus efficace quand les microtubules sont acétylés: les moteurs impliqués, de la famille des kinésines et des dynéines ${ }^{1}$, ont en effet plus d'affinité pour les microtubules acétylés. L'acétylation de la lysine 40 de la tubuline est par ailleurs à l'origine d'un affaiblissement des interactions latérales entre protofilaments [15]. Ceci pourrait donc expliquer l'influence du taux d'acétylation de la tubuline sur le nombre de protofilaments d'un microtubule [16]. Cette diminution de la force des interactions latérales augmente aussi la résistance des microtubules aux déformations mécaniques répétées, assurant ainsi la persistance des microtubules à longue durée de vie [15].

\section{Le cycle de détyrosination/retyrosination et ses enzymes}

La tyrosine C-terminale de la tubuline $\alpha$ peut être hydrolysée par la tubuline carboxypeptidase (TCP) et ré-additionnée par la tubuline tyrosine ligase (TTL). À la différence de l'acétylation, la réaction de tyrosination est spécifique de la tubuline, elle n'est observée pour aucune autre protéine connue. Le cycle enzymatique généré par l'action de la TCP et de la TTL est conservé chez les eucaryotes supérieurs. Découvert en 1972, le cycle de détyrosination/retyrosination (détyr/ retyr) est la toute première description d'une modification post-traductionnelle spécifique de la tubuline [17] (figure 3B).

Peu de temps après cette découverte, une forme de tubuline résistante in vitro à la retyrosination a été décrite. Cette tubuline non-tyrosinable représente près d'un tiers de la tubuline cérébrale chez le rat adulte [18, 19]. Sa nature moléculaire a été élucidée en 1991 [20]. Elle diffère de la sous-unité $\alpha$ tyrosinée par la suppression des deux acides aminés $C$-terminaux sous l'action des carboxypeptidases cytosoliques (CCP). Cette modification du site d'action de la TTL explique pourquoi cette forme de tubuline ne peut pas être tyrosinée. Elle a été baptisée «tubuline $\Delta 2 »[20,21]$. Dans les tissus neuronaux, une forme supplémentaire de tubuline, la «tubuline $\Delta 3 »$, correspondant à l' $\alpha$-tubuline dépourvue des 3 derniers acides aminés, a également été décrite ; elle est plus abondante dans le cerveau embryonnaire que dans le cerveau adulte, ce qui suggère qu'elle est importante au cours du développement [18].

\section{La tubuline tyrosine ligase}

La tubuline tyrosine ligase (TTL) a été purifiée pour la première fois en 1985, grâce à un anticorps monoclonal spécifique de la protéine native. Chez la souris, l'invalidation du gène codant la TTL entraîne la mort périnatale des souriceaux, soulignant le rôle vital de cette protéine et du cycle de détyr/retyr [22]. La résolution de la structure 3D de la TTL a permis de comprendre pourquoi la réaction de tyrosination se fait sur les dimères de tubuline libres et non sur les microtubules. $\varepsilon n$ effet, l'interaction entre la TTL et la tubuline met en jeu un site de la tubuline qui n'est plus exposé lorsqu'elle est assemblée en micro-

${ }^{1}$ Les kinésines et les dynéines sont des protéines motrices, qui se déplacent le long des microtubules. Les kinésines se déplacent vers l'extrémité plus et les dynéines vers l'extrémité moins. tubules [23]. Le fait que la TTL soit sélective de la tubuline s'expliquerait par son interaction simultanée avec les sous-unités $\alpha$ et $\beta$ du dimère. L'activité de la TTL apparaît être modulée par sa phosphorylation: des formes mutantes phospho-mimétiques suggèrent en effet que la phosphorylation de la sérine 152 bloque l'activité de l'enzyme [23]. Un anticorps monoclonal capable d'inhiber la TTL a été décrit [24] et des composés de la classe des sesterterpènes se sont révélés prometteurs pour le développement futur d'inhibiteurs pharmacologiques spécifiques de cette enzyme [25].

\section{La tubuline carboxypeptidase}

Bien que de nombreux essais de purification aient été tentés, la nature de la TCP est restée inconnue jusque récemment, lorsque deux équipes ont réussi à montrer simultanément, et avec deux approches différentes, qu'un complexe entre des tubuline carboxypeptidases, les vasohibines et les small vasohibin-binding protein (ou complexe VASH/SVBP) était $(\rightarrow)$ Voir la Nouvelle de M.J. Moutin et al., page 1022 de ce numéro peptidase de la tubuline $[26,27,66](\rightarrow)$.

Différents inhibiteurs pharmacologiques de l'activité TCP ont été décrits: un inhibiteur peptidomimétique covalent ainsi que le parthénolide ${ }^{2}$ et le TPCK (tolylsulfonylphenylalanyl chloromethyl ketone) [26, 28, 29]. Ces deux derniers agents sont peu sélectifs de la TCP.

\section{Fonctions moléculaires du cycle de détyrosination/retyrosination}

Au sein du microtubule, l'extrémité C-terminale de la tubuline $\alpha$ n'est pas impliquée dans les interactions entre les sous-unités de tubuline, mais se déploie dans le cytoplasme à partir de la paroi des microtubules. C'est pourquoi il a été rapidement suspecté que cette extrémité de la protéine était impliquée dans des interactions avec des protéines régulatrices des microtubules. Effectivement, la présence de la tyrosine C-terminale conditionne la liaison de protéines de type CAP-Gly (cytoskeleton-associated protein-glycine-rich domain) à l'extrémité «plus » des microtubules [30]. Par ailleurs, elle est indispensable au fonctionnement efficace des moteurs dépolymérisants de la famille de la kinésine-13 [31]. En revanche, la tubuline sous sa forme détyrosinée favorise la liaison et l'activité processive de la kinésine $5 C$ et de CENP- $\varepsilon$ (kinésine 7 ) $[29,32]$.

\footnotetext{
${ }^{2}$ Une lactone sesquiterpénique.
} 
A

\begin{tabular}{|c|c|c|c|c|}
\hline Enzyme & UniProt & Nom & Taille & Inhibiteur \\
\hline Acétyltransférase & Q5SQI0 & $\begin{array}{c}\text { aTATl } \\
\text { (alpha tubulin acetyltransferase 1) } \\
\text { Mec-17 homologue }\end{array}$ & 421 aa & $/$ \\
\hline
\end{tabular}

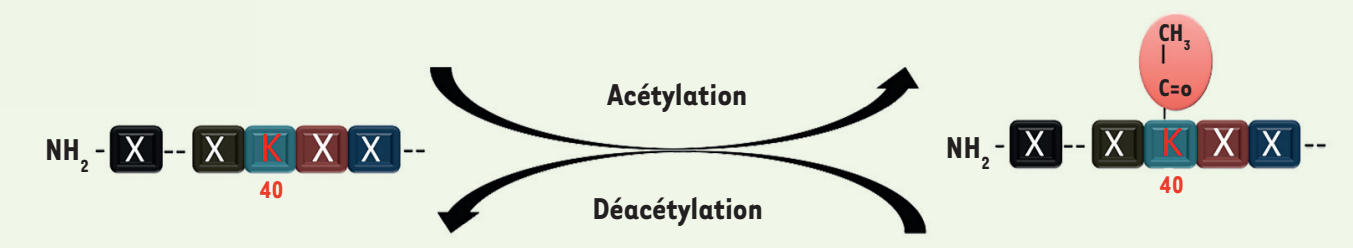

\begin{tabular}{|c|c|c|c|c|}
\hline Enzyme & UniProt & Nom & Taille & Inhibiteur \\
\hline \multirow{4}{*}{ Déacétylase } & Q9UBN7 & HDAC6 (histone deacetylase 6) & 1215 aa & $\begin{array}{c}\text { Trichostatine A } \\
\text { MC 1568 } \\
\text { Tubastatine A } \\
\text { Tubacine }\end{array}$ \\
\cline { 2 - 5 } & & & & $\begin{array}{c}\text { Sirtinol } \\
\text { Nicotinamide } \\
\end{array}$ \\
\cline { 2 - 5 } & Q81XJ6 & Sirt2 (Sirtuin 2) & 389 aa & $\begin{array}{c}\text { B2 } \\
\text { AGK2 } \\
\end{array}$ \\
& & & & BML-266 \\
& & & AK-1 \\
\hline
\end{tabular}

B

\begin{tabular}{|c|c|c|c|c|}
\hline Enzyme & UniProt & Nom & Taille & Inhibiteur \\
\hline \multirow{2}{*}{$\begin{array}{l}\text { Carboxypeptidase } \\
\text { Sous-unité } \\
\text { catalytique }\end{array}$} & Q7L8A9 & VASHI (vasohibine-1) & 365 a a & Epoy \\
\hline & Q86V25 & VASH2 (vasohibine-2) & 355 a a & \multirow{2}{*}{$\begin{array}{l}\text { Parthénolide } \\
\text { TPCK }\end{array}$} \\
\hline $\begin{array}{l}\text { Sous-unité } \\
\text { régulatrice }\end{array}$ & Q8N300 & $\begin{array}{l}\text { SVBP (small vasohibin-binding } \\
\text { protein) }\end{array}$ & $66 \mathrm{aa}$ & \\
\hline
\end{tabular}

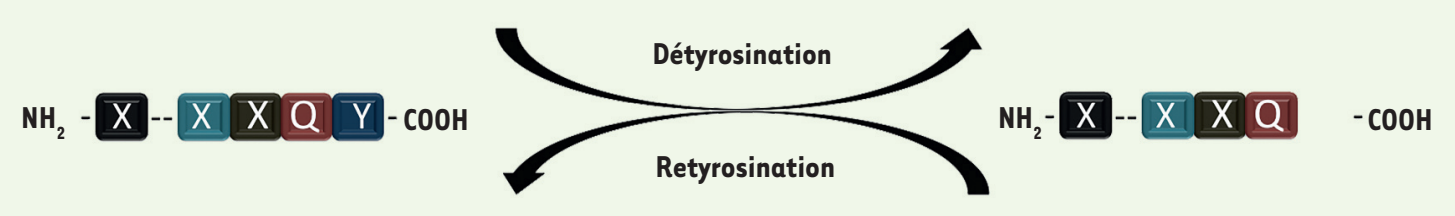

\begin{tabular}{|c|c|c|c|c|}
\hline Enzyme & UniProt & nom & Taille & Inhibiteur \\
\hline $\begin{array}{c}\text { Tyrosine } \\
\text { ligase }\end{array}$ & 08NG68 & TTL (tubulin tyrosine ligase) & 377 aa & $\begin{array}{c}\text { Sesterterpènes } \\
\text { Anticorps bloquant }\end{array}$ \\
\hline
\end{tabular}

Figure 3. Les acteurs de la (dé)acétylation et du cycle détyr/retyr. A. La (dé)acétylation, ses enzymes et ses inhibiteurs. B. Le cycle de détyrosination/retyrosination, ses enzymes et ses inhibiteurs.

Dérégulations de la (dé)acétylation ou du cycle de détyrosination/ retyrosination des microtubules dans les pathologies

\section{Acétylation et pathologies neuronales}

La maladie de Charcot-Marie-Tooth est une pathologie héréditaire du système nerveux périphérique. Elle peut être accompagnée d'une diminution de l'acétylation de la tubuline et d'une altération de l'influx nerveux axonal. Dans un modèle murin de la maladie, l'inhibition de la déacétylase HDAC6 rétablit le phénotype sauvage [33]. Par ailleurs, une diminution de l'acétylation des microtubules a été détectée dans les neurones des patients atteints de 
la maladie d'Alzheimer. Cette observation corrèle avec un défaut de transport et une accumulation de protéines de conformation aberrante qui induit une dégénérescence synaptique [34]. Le peptide amyloïde $A \beta$ de l'APP (amyloid precursor protein) semble être responsable de cette baisse d'acétylation. Dans un modèle murin de la maladie d'Alzheimer, la réduction d'expression d'HDAC6, ou l'inhibition de son activité, peut ainsi améliorer les défauts cognitifs et de mémoire, en augmentant l'acétylation des microtubules. Le mécanisme moléculaire responsable pourrait être une amélioration du transport neuronal par les moteurs quand les microtubules sont acétylés, comme cela a été décrit pour la maladie de Huntington; le défaut de transport vésiculaire dans la maladie de Huntington est en effet amélioré après inhibition de I'HDAC6 [35]. Néanmoins, ces résultats n'ont pas été confirmés in vivo dans un modèle murin de la maladie de Huntington dans lequel le gène codant HDAC6 a été invalidé.

Un rôle de l'acétylation des microtubules dans la maladie de Parkinson a aussi été décrit, mais des résultats contradictoires ont été obtenus selon les modèles utilisés. Ainsi, la kinase LRRK2 (leucine-rich repeat kinase 2) portant une mutation pathogénique observée dans la maladie de Parkinson interagit préférentiellement avec les microtubules non-acétylés pour bloquer le transport axonal, ce qui pourrait jouer un rôle dans l'établissement de la maladie. Ce phénomène peut être inversé par l'inhibition, ou la diminution d'expression, par knock-down, des déacétylases HDAC6 ou Sirt2 [36]. Dans un autre modèle, utilisant la 6-hydroxydopamine pour détruire sélectivement les neurones de la substance noire, une altération de la dynamique des microtubules, due à une diminution de l'activité de la déacétylase Sirt2, est observée. Ces défauts peuvent être corrigés par la restauration de l'activité de cette déacétylase [37].

\section{Cycle de détyrosination/retyrosination et pathologies neuronales}

À l'heure actuelle, aucune pathologie neuronale résultant spécifiquement d'une dérégulation du cycle détyr/retyr n'a été décrite. Néanmoins, une désorganisation des réseaux neuronaux est observée chez des souriceaux invalidés pour le gène codant la TTL. Elle serait à l'origine de leur mort périnatale [22]. Une diminution de l'expression du complexe VASH/SVBP, qui induit une baisse de détyrosination, perturbe la différenciation et la migration des neurones [26]. Un défaut de migration du cône de croissance pourrait être impliqué. En effet, l'état tyrosiné de la tubuline est indispensable aux réarrangements des microtubules et des filaments d'actine nécessaires aux mouvements corrects du cône de croissance au cours du développement [38]. De plus, il a été montré que la tubuline tyrosinée agit comme un indicateur directionnel pour la kinésine-l et que ce mécanisme est requis pour le maintien de la polarité neuronale [39]. Dans un modèle murin, dans lequel la kinase GSK3 (glycogen synthase kinase 3) est constitutivement active, la repousse des neurones après blessure du nerf sciatique est accélérée, ce qui conduit à une amélioration motrice et sensitive. Un des mécanismes sous-jacents mettrait en jeu une accélération de la dynamique des microtubules grâce à la phosphorylation de MAPIB (microtubule-associated protein $1 B$ ) et à la présence d'une plus grande proportion de microtubules tyrosinés. Une inhibition de la détyrosination, par un traitement avec le parthénolide, accélèrerait la régénération axonale chez les souris sauvages. L'inhibition de l'activité TCP pourrait donc représenter une piste thérapeutique pour le traitement des lésions nerveuses [40].

\section{Acétylation et cancer}

La transition épithélio-mésenchymateuse (EMT) est considérée comme une prémisse de la transformation des cellules différenciées vers une dédifférenciation et un état cancéreux. Une diminution de l'acétylation de l' $\alpha$-tubuline est observée lors de la transition épithélio-mésenchymateuse induite par le TGF- $\beta$ (transforming growth factor- $\beta$ ). Cette transition peut être bloquée par l'inhibition de l'HDAC6 [41]. Cependant, une augmentation de l'acétylation des microtubules est observée dans la plupart des tumeurs. Par exemple, l'acétylation de la tubuline est élevée dans les cancers du sein et fortement corrélée à un mauvais pronostic [42]. Une surexpression d'ATATl et I'hyper-acétylation de l' $\alpha$-tubuline qui en résulte sont également décrites dans les cancers du côlon. L'excès d'acétylation favoriserait la prolifération et les capacités invasives des cellules [43]. Une augmentation de l'acétylation de la tubuline est également détectée dans certains carcinomes squameux de la tête et du cou [44].

Un aspect important à souligner est que le suppresseur de tumeurs Merlin n'est pas actif si les microtubules sont déacétylés [45]. CYLD (ubiquitin carboxyl-terminal hydrolase), un autre suppresseur de tumeurs, interagit avec HDAC6 et l'inhibe, induisant une augmentation de la tubuline acétylée autour du noyau et dans le corps intermédiaire, ce qui ralentit la mitose et la cytocinèse [46]. Plusieurs études ont montré que les drogues qui ciblent HDAC6 augmentaient l'acétylation des microtubules et inhibaient la prolifération des cellules leucémiques et des cellules cancéreuses des tumeurs solides [47, 48].

Comment réconcilier ces données révélant, d'une part, une augmentation de la tubuline acétylée dans les cancers et d'autre part, un effet bénéfique d'une hyper-acétylation de la tubuline? Une explication possible est que l'augmentation de l'acétylation de la tubuline est une tentative de la cellule pour se défendre contre la transformation tumorale. L'approche thérapeutique visant à inhiber I'HDAC6 renforcerait ce mécanisme de défense. L'hyper-acétylation de la lysine 40 pourrait empêcher sa triméthylation par SETD2 (SET domain containing 2) dans les microtubules du fuseau mitotique. Cette méthylation est nécessaire pour l'accomplissement d'une cytocinèse correcte [13]. Ceci pourrait être un argument en faveur de l'intérêt d'inhiber les tubuline déacétylases pour réduire la prolifération des cellules cancéreuses. 
Implication du cycle de détyrosination-retyrosination

\section{dans les processus cancéreux}

Il a été observé, initialement dans les cancers du sein [49], puis dans d'autres types de cancers [50], que la TTL est éliminée au cours de la croissance tumorale, se comportant ainsi comme un suppresseur de tumeur. La perte de TTL se traduit par une accumulation anormale de tubuline détyrosinée, normalement minoritaire dans les cellules épithéliales. Dans une série de 134 tumeurs du sein, la présence de tubuline abondamment détyrosinée a été observée dans la moitié des cas. Cette détyrosination massive est révélée presque exclusivement dans les cancers ayant un grade histo-pronostique de Scarff Bloom et Richardson ${ }^{3}$ maximal [51]. La détyrosination de la tubuline a donc une signification pronostique propre, indépendamment des facteurs pronostiques habituellement pris en compte en clinique.

Différents travaux ont été menés pour déterminer le lien entre détyrosination de la tubuline et invasion tumorale. La tubuline détyrosinée est enrichie dans des protrusions membranaires, les microtentacules, présentes sur les cellules circulantes de cancer du sein [52]. Ces microtentacules contribuent au processus métastatique. Elles permettent aux cellules qui se sont échappées des tumeurs primaires de s'attacher aux parois de petits vaisseaux sanguins pour faciliter leur extravasation et, ensuite, la formation de métastases. Le parthénolide qui exerce une action inhibitrice sur la TCP, réduit la formation de ces microtentacules et empêche l'attachement des cellules tumorales aux cellules endothéliales. Par ailleurs, un équilibre précis entre microtubules tyrosinés et microtubules détyrosinés est important pour le bon déroulement de la mitose. La détyrosination des microtubules permet leur interaction sélective avec la kinésine-7, responsable du guidage correct des chromosomes à l'équateur de la cellule lors de la mitose [29].

Puisque la TTL se comporte comme un suppresseur de tumeur, il est logique de s'interroger sur le rôle oncogénique éventuel de la TCP, et donc de l'expression des protéines VASH 1 et 2, ainsi que de SVBP, dans les tumeurs. Les protéines VASHl/2 ont été identifiées en 2004 par un groupe japonais [53], ignorant qu'il s'agissait d'enzymes en lien avec le cytosquelette. Initialement décrites comme le produit de gènes dont l'expression est induite par le VEGF (vascular endothelial growth factor), on pensait qu'il s'agissait de protéines sécrétées, régulatrices de l'angiogenèse, avec un mécanisme de sécrétion mal compris. En raison de leur rôle potentiel dans la régulation de l'angiogenèse, l'expression de VASHI/2 a été analysée dans différents types de cancer, toujours par des équipes japonaises [54]. À l'exception du cancer du côlon, l'expression élevée de VASHl/2 s'est avérée toujours corrélée à un grade histologique élevé et/ou à un mauvais pronostic et le niveau d'expression de VASHl peut être utilisé pour prédire la réponse au traitement thérapeutique de patientes atteintes de cancer du sein [55]. L'ensemble de ces résultats suggère que - plutôt que la perte de TTL ou l'augmentation de l'expression de VASHl/2 - l'augmentation de tubuline détyrosinée qui en résulte pourrait avoir un rôle dans la progression tumorale.

${ }^{3}$ La classification de Scarff, Bloom et Richardson est fondée sur trois critères histologiques différents : le degré de différenciation architecturale, le nombre de mitoses par champ et l'importance du pléiomorphisme nucléaire.
Modifications de la tubuline et propagation virale Une stratégie utilisée par certains virus comme le virus de l'immunodéficience humaine (VIH) pour se propager est de cibler les protéines de l'extrémité «plus » des microtubules pour provoquer leur stabilisation. Les microtubules ainsi stabilisés sont alors détyrosinés et acétylés et sont reconnus par des protéines motrices spécifiques qui facilitent le transport des particules virales vers le noyau [56]. Cibler les enzymes responsables de ces modifications post-traductionnelles pourrait donc perturber l'interaction des protéines motrices [57] et représenter une stratégie intéressante pour réduire l'infection virale. En accord avec cette hypothèse, il a été montré qu'une inhibition d'HDAC6 potentialise les traitements de cancers résultant d'une infection oncolytique virale [58].

\section{Acétylation, détyrosination et pathologies cardio- vasculaires}

Les troubles du rythme cardiaque, comme la fibrillation atriale, ont pour origine un dysfonctionnement de la contraction des cardiomyocytes souvent accompagné d'une déacétylation de l' $\alpha$-tubuline. L'inhibition de la déacétylase HDAC6 empêche ces altérations des cardiomyocytes et la progression des symptômes pathologiques [59]. D'autres pathologies cardiaques ont pour origine une accumulation de protéines mal repliées. Leur élimination par autophagie est nécessaire pour améliorer la fonction cardiaque. Or l'inhibition d'HDAC6, qui provoque une hyper-acétylation des microtubules, induit une stimulation de l'autophagie [60]. Enfin, dans un modèle d'ischémie cérébrale chez le rat, la perte des cellules neuronales peut être réduite par un traitement post-ischémique avec la Tubastatine A, un inhibiteur d'HDAC6 [61].

L'état de tyrosination des microtubules représente un signal de régulation important de la mécanotransduction [62]. Dans le myocarde, quand les cellules cardiaques se contractent, les microtubules résistent à la compression en fléchissant, prenant alors une allure de sinusoïde. La détyrosination des microtubules favorise leur interaction avec la desmine des sarcomères, ce qui confère aux myocytes une résistance mécanique. Un excès de détyrosination peut ainsi participer à la diminution de la fonction cardiaque dans certains états pathologiques [63]. Dans les muscles squelettiques, la détyrosination de l' $\alpha$-tubuline augmente également la rigidité du cytosquelette et la mécanotransduction. Ainsi, dans un modèle murin de dystrophie musculaire, l'inhibition de la TCP par le parthénolide corrige la détyrosination aberrante de l' $\alpha$-tubuline et empêche les arythmies et les blessures du muscle squelettique provoquées par des contractions accrues [64]. 


\section{Conclusion}

Des données de plus en plus nombreuses indiquent que les modifications de la tubuline sont impliquées dans différentes pathologies. La découverte récente de l'activité tubuline carboxypeptidase du complexe vasohibine/SVBP permet désormais de décrire l'entièreté du cycle détyr/retyr. Cela ouvre la voie au développement de modèles animaux, dont l'analyse devrait encore réserver des surprises. Les enzymes de la (dé)acétylation sont connues depuis plus longtemps et les modèles murins sont déjà développés. On sait désormais qu'en plus de ces enzymes, il en existe d'autres présentant une activité similaire, comme Sirt2. Des substrats autres que la tubuline ont aussi été décrits pour les enzymes impliquées dans la (dé)acétylation.

En ce qui concerne le cycle détyr/retyr, si la TTL présente une spécificité pour la tubuline, la possibilité de l'existence d'autres substrats pour VASH/SVBP reste à examiner. L'existence d'autres enzymes présentant une activité tubuline carboxypeptidase peut aussi être envisagée. Ces connaissances sont importantes pour le développement de nouveaux inhibiteurs pharmacologiques sélectifs. $\diamond$

\section{SUMMARY}

On the road to deciphering the tubulin code: focus on acetylation and detyrosination

Microtubules are cytoskeletal fibers formed by the assembly of $\alpha$ - and $\beta$-tubulin heterodimers. They contribute to cell morphology, mobility and polarity, as well as to cellular transport processes and cell division. The microtubular network constantly adapts to cellular needs and may be composed of very dynamic or more stable microtubules. To regulate their diverse functions in a spatio-temporal manner, microtubules are subjected to numerous reversible post-translational modifications, which generate the "tubulin code". This review focuses on two modifications characteristic of stable microtubules - acetylation and detyrosination of $\alpha$-tubulin - and their deregulation in certain pathologies. $\diamond$

\section{LIENS D'INTÉRÊT}

Les auteurs déclarent n'avoir aucun lien d'intérêt concernant les données publiées dans cet article.

\section{RÉFÉRENCES}

1. Sadoul K, Khochbin $\mathrm{S}$. The growing landscape of tubulin acetylation: lysine 40 and many more. Biochem J 2016 ; $473: 1859-68$.

2. Gadadhar S, Bodakuntla S, Natarajan K, Janke C. The tubulin code at a glance. J Cell Sci 2017 ; $130: 1347-53$.

3. L'Hernault SW, Rosenbaum JL. Chlamydomonas alpha-tubulin is posttranslationally modified by acetylation on the epsilon-amino group of a lysine. Biochemistry $1985 ; 24: 473-8$

4. Akella JS, Wloga D, Kim J, et al. MEC-17 is an alpha-tubulin acetyltransferase. Nature $2010 ; 467$ : 218-22.

5. Nogales $\varepsilon$, Whittaker M, Milligan RA, Downing KH. High-resolution model of the microtubule. Cell $1999 ; 96: 79-88$.

6. Szyk A, Deaconescu AM, Spector J, et al. Molecular basis for age-dependent microtubule acetylation by tubulin acetyltransferase. Cell $2014 ; 157$ : 1405-15.

7. Coombes C, Yamamoto A, McClellan M, et al. Mechanism of microtubule lumen entry for the alphatubulin acetyltransferase enzyme alphaTATl. Proc Natl Acad Sci U S A 2016 ; 113 : ع7176-ع84.

8. Montagnac $G$, Chavrier P. Quand les microtubules rencontrent les puits recouverts de clathrine et permettent aux cellules de tenir le cap. Med Sci (Paris) 2014 ; $30: 130$-3.

9. Hubbert C, Guardiola A, Shao R, et al. HDAC6 is a microtubule-associated deacetylase. Nature $2002 ; 417: 455-8$.
10. North BJ, Marshall BL, Borra MT, et al. The human Sir2 ortholog, SIRT2, is an NAD+-dependent tubulin deacetylase. Mol Cell $2003 ; 11: 437-44$.

11. Skultetyova L, Ustinova K, Kutil Z, et al. Human histone deacetylase 6 shows strong preference for tubulin dimers over assembled microtubules. Sci Rep $2017 ; 7: 11547$.

12. Skoge RH, Ziegler M. SIRT2 inactivation reveals a subset of hyperacetylated perinuclear microtubules inaccessible to HDAC6. J Cell Sci 2016 ; 129 : 297282.

13. Park IY, Powell RT, Tripathi DN, et al. Dual Chromatin and Cytoskeletal Remodeling by SETD2. Cell $2016 ; 166: 950-62$.

14. Alonso VL, Ritagliati C, Cribb P, et al. Overexpression of bromodomain factor 3 in Trypanosoma cruzi (TcBDF3) affects differentiation of the parasite and protects it against bromodomain inhibitors. FEBS J 2016 ; 283 : 2051-66.

15. Portran D, Schaedel L, Xu Z, et al. Tubulin acetylation protects long-lived microtubules against mechanical ageing. Nat Cell Biol $2017 ; 19$ : 391-8.

16. Howes SC, Alushin GM, Shida T, et al. Effects of tubulin acetylation and tubulin acetyltransferase binding on microtubule structure. Mol Biol Cell $2014 ; 25: 257-66$.

17. Barra HS, Unates LE, Sayavedra MS, Caputto R. Capacities for binding amino acids by tRNAs from rat brain and their changes during development.J Neurochem $1972 ; 19: 2289-97$.

18. Aillaud C, Bosc C, Saoudi Y, et al. Evidence for new C-terminally truncated variants of alpha- and beta-tubulins. Mol Biol Cell $2016 ; 27: 640-53$.

19. Paturle-Lafanechere L, Manier M, Trigault N, et al. Accumulation of delta 2-tubulin, a major tubulin variant that cannot be tyrosinated, in neuronal tissues and in stable microtubule assemblies. J Cell Sci 1994 ; 107 : 1529-43.

20. Paturle-Lafanechere $L$, Edde $B$, Denoulet $P$, et al. Characterization of a major brain tubulin variant which cannot be tyrosinated. Biochemistry $1991 ; 30: 10523-8$.

21. Lafanechere L, Job D. The third tubulin pool. Neurochem Res $2000 ; 25: 11-8$.

22. Erck C, Peris L, Andrieux A, et al. A vital role of tubulin-tyrosine-ligase for neuronal organization. Proc Natl Acad Sci U S A 2005 ; 102 : 7853-8.

23. Prota AE, Magiera MM, Kuijpers $M$, et al. Structural basis of tubulin tyrosination by tubulin tyrosine ligase. J Cell Biol $2013 ; 200: 259-70$.

24. Wehland J, Weber K. Tubulin-tyrosine ligase has a binding site on beta-tubulin: a two-domain structure of the enzyme. J Cell Biol $1987 ; 104$ : 1059-67.

25. Dal Piaz F, Vassallo A, Lepore L, et al. Sesterterpenes as tubulin tyrosine ligase inhibitors. First insight of structure-activity relationships and discovery of new lead. J Med Chem 2009 ; 52 : 3814-28.

26. Aillaud C, Bosc C, Peris L, et al. Vasohibins/SVBP are tubulin carboxypeptidases (TCPs) that regulate neuron differentiation. Science $2017 ; 358: 1448-53$.

27. Nieuwenhuis J, Adamopoulos A, Bleijerveld OB, et al. Vasohibins encode tubulin detyrosinating activity. Science $2017 ; 358: 1453-56$.

28. Fonrose $X$, Ausseil F, Soleilhac $\varepsilon$, et al. Parthenolide inhibits tubulin carboxypeptidase activity. Cancer Res 2007 ; 67 : 3371-8.

29. Barisic M, Silva e Sousa R, Tripathy SK, et al. Mitosis. Microtubule detyrosination guides chromosomes during mitosis. Science 2015 ; 348 : 799-803.

30. Peris L, Thery M, Faure J, et al. Tubulin tyrosination is a major factor affecting the recruitment of CAP-Gly proteins at microtubule plus ends.J Cell Biol $2006 ; 174: 839-49$.

31. Peris L, Wagenbach M, Lafanechere L, et al. Motor-dependent microtubule disassembly driven by tubulin tyrosination. J Cell Biol 2009; 185 : 1159-66.

32. Dunn S, Morrison દE, Liverpool TB, et al. Differential trafficking of Kif5c on tyrosinated and detyrosinated microtubules in live cells. J Cell Sci 2008 ; $121: 1085-95$.

33. d'ydewalle C, Krishnan J, Chiheb DM, et al. HDAC6 inhibitors reverse axonal loss in a mouse model of mutant HSPBI-induced Charcot-Marie-Tooth disease. Nat Med $2011 ; 17$ : 968-74.

34. Butler D, Bendiske J, Michaelis ML, et al. Microtubule-stabilizing agent prevents protein accumulation-induced loss of synaptic markers. Eur J Pharmacol $2007 ; 562: 20-7$.

35. Dompierre JP, Godin JD, Charrin BC, et al. Histone deacetylase 6 inhibition compensates for the transport deficit in Huntington's disease by increasing tubulin acetylation. J Neurosci $2007 ; 27: 3571-83$.

36. Godena VK, Brookes-Hocking N, Moller A, et al. Increasing microtubule acetylation rescues axonal transport and locomotor deficits caused by LRRK2 Roc-COR domain mutations. Nat Commun 2014 ; 5 : 5245.

37. Patel VP, Chu CT. Decreased SIRT2 activity leads to altered microtubule dynamics in oxidatively-stressed neuronal cells: implications for Parkinson's disease. Exp Neurol 2014 ; 257 : 170-81. 


\section{RÉFÉRENCES}

38. Marcos S, Moreau J, Backer S, et al. Tubulin tyrosination is required for the proper organization and pathfinding of the growth cone. PLoS One $2009 ; 4$ : e5405.

39. Konishi Y, Setou M. Tubulin tyrosination navigates the kinesin-1 motor domain to axons. Nat Neurosci $2009 ; 12: 559-67$

40. Gobrecht P, Andreadaki A, Diekmann H, et al. Promotion of functional nerve regeneration by inhibition of microtubule detyrosination. J Neurosci $2016 ; 36: 3890-902$

41. Gu S, Liu Y, Zhu B, et al. Loss of alpha-tubulin acetylation is associated with TGF-beta-induced epithelial-mesenchymal transition. J Biol Chem 2016 ; 291 : 5396-405.

42. Boggs AE, Vitolo MI, Whipple RA, et al. Alpha-tubulin acetylation elevated in metastatic and basal-like breast cancer cells promotes microtentacle formation, adhesion, and invasive migration. Cancer Res $2015 ; 75: 203-15$.

43. Oh $S$, You $\varepsilon$, Ko $P$, et al. Genetic disruption of tubulin acetyltransferase, alphaTATl, inhibits proliferation and invasion of colon cancer cells through decreases in Wntl/beta-catenin signaling. Biochem Biophys Res Commun 2017 ; 482 : 8-14.

44. Saba NF, Magliocca KR, Kim S, et al. Acetylated tubulin (AT) as a prognostic marker in squamous cell carcinoma of the head and neck. Head Neck Pathol $2014 ; 8$ : 66-72.

45. Aguilar A, Becker L, Tedeschi T, et al. Alpha-tubulin $K 40$ acetylation is required for contact inhibition of proliferation and cell-substrate adhesion. Mol Biol Cell $2014 ; 25$ : 1854-66.

46. Wickstrom SA, Masoumi KC, Khochbin S, et al. CYLD negatively regulates cell-cycle progression by inactivating HDAC6 and increasing the levels of acetylated tubulin. EMBO J 2010 ; 29 : 131-44.

47. Aldana-Masangkay GI, Rodriguez-Gonzalez A, Lin T, et al. Tubacin suppresses proliferation and induces apoptosis of acute lymphoblastic leukemia cells. Leuk Lymphoma 2011 ; 52 : 1544-55.

48. Lee JH, Mahendran A, Yao Y, et al. Development of a histone deacetylase 6 inhibitor and its biological effects. Proc Natl Acad Sci U S A 2013 ; 110 : 15704-9.

49. Lafanechere L, Courtay-Cahen C, Kawakami T, et al. Suppression of tubulin tyrosine ligase during tumor growth. J Cell Sci $1998 ; 111$ ( Pt 2) : 171-81.

50. Kato C, Miyazaki K, Nakagawa A, et al. Low expression of human tubulin tyrosine ligase and suppressed tubulin tyrosination/detyrosination cycle are associated with impaired neurona differentiation in neuroblastomas with poor prognosis. Int J Cancer 2004 ; $112: 365-75$.

51. Mialhe A, Lafanechere L, Treilleux I, et al. Tubulin detyrosination is a frequent occurrence in breast cancers of poor prognosis. Cancer Res $2001 ; 61: 5024-7$.

52. Whipple RA, Matrone MA, Cho EH, et al. Epithelial-to-mesenchymal transition promotes tubulin detyrosination and microtentacles that enhance endothelial engagement. Cancer Res $2010 ; 70$ : 8127-37.

53. Watanabe K, Hasegawa $Y$, Yamashita $\mathrm{H}$, et al. Vasohibin as an endothelium-derived negative feedback regulator of angiogenesis. J Clin Invest 2004 ; 114 : 898-907.

54. Kobayashi H, Kosaka T, Mikami S, et al. Vasohibin-1 as a novel microenvironmental biomarker for patient risk reclassification in low-risk prostate cancer. Oncotarget $2018 ; 9: 10203-10$.
55. Kapoor S. Comment and reply on: Vasohibin-l and its emerging role in the evolution and progression of systemic tumors besides renal cell carcinomas. Expert Opin Ther Targets $2013 ; 17: 105-6$.

56. Sabo Y, Walsh D, Barry DS, et al. HIV-1 induces the formation of stable microtubules to enhance early infection. Cell Host Microbe $2013 ; 14$ : 535 46.

57. Zhou J, Scherer J, Yi J, Vallee RB. Role of kinesins in directed adenovirus transport and cytoplasmic exploration. PLoS Pathog 2018 ; 14 : e1007055.

58. Nakashima H, Kaufmann JK, Wang Py, et al. Histone deacetylase 6 inhibition enhances oncolytic viral replication in glioma. J Clin Invest 2015; 125 : 4269-80.

59. Zhang D, Wu CT, Qi X, et al. Activation of histone deacetylase- 6 induces contractile dysfunction through derailment of alpha-tubulin proteostasis in experimental and human atrial fibrillation. Circulation $2014 ; 129$ : 346-58.

60. McLendon PM, Ferguson BS, Osinska $\mathrm{H}$, et al. Tubulin hyperacetylation is adaptive in cardiac proteotoxicity by promoting autophagy. Proc Natl Acad Sci U S A 2014 ; 111 : ع5178-86.

61. Wang Z, Leng $Y$, Wang J, et al. Tubastatin A, an HDAC6 inhibitor, alleviates stroke-induced brain infarction and functional deficits: potential roles of alpha-tubulin acetylation and FGF-21 up-regulation. Sci Rep 2016; 6 : 19626.

62. Belmadani S, Pous C, Fischmeister R, Mery PF. Post-translational modifications of tubulin and microtubule stability in adult rat ventricular myocytes and immortalized HL-1 cardiomyocytes. Mol Cell Biochem 2004 ; $258: 35-48$.

63. Robison P, Caporizzo MA, Ahmadzadeh H, et al. Detyrosinated microtubules buckle and bear load in contracting cardiomyocytes. Science 2016; 352 : aaf0659.

64. Kerr JP, Robison P, Shi G, et al. Detyrosinated microtubules modulate mechanotransduction in heart and skeletal muscle. Nat Commun $2015 ; 6$ : 8526.

65. Ran J, Yang Y, Li D, et al. Deacetylation of alpha-tubulin and cortactin is required for HDAC6 to trigger ciliary disassembly. Sci Rep $2015 ; 5: 12917$.

66. Moutin MJ, Bosc C, Peris L, Andrieux A. La boucle est bouclée : des complexes enzymatiques qui détyrosinent les microtubules enfin découverts. Med Sci (Paris) $2018 ; 34: 1022-5$

TIRÉS À PART

K. Sadoul

Bon de commande à retourner à EDP Sciences, 17 avenue du Hoggar, 91944 Les Ulis Cedex Tél. : 0149856069 - Fax : 0149850345 - E-mail : francois.flori@edpsciences.org

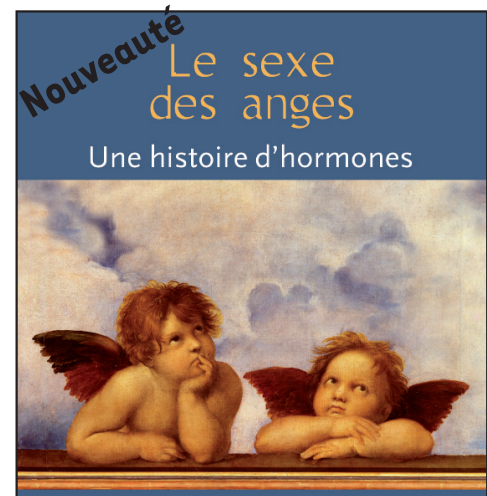

NATHAL E JOSSO IlLustrations MarTine Netter
NOM : Prénom :

Adresse :

Code postal : Ville :

Pays :

Fonction :

Je souhaite recevoir l'ouvrage

Le sexe des anges : $20 €+3 €$ de port $=23 €$ TTC

en

exemplaire, soit un total de

Par chèque, à l'ordre de EDP Sciences

Par carte bancaire

$\square$ Visa

$\square$ Eurocard/Mastercard

Carte $n^{\circ}$
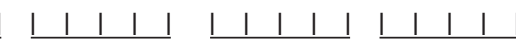

Signature :

Date d'expiration :

$\mathrm{N}^{\circ}$ de contrôle au dos de la carte :

iil Inserm 